\title{
Depresión en parados. El efecto de la resiliencia y el apoyo social
}

\author{
$M^{a}$ PILAR Sospedra Sospedra \\ mapisospedra@yahoo.com \\ NASTRA ARES ARMERO \\ nastracs@gmail.com \\ Ma PILAR JARA JIMÉNEZ \\ jara@uji.es
}

\section{Resumen}

Introducción: La actualidad nos pone ante una situación de crisis en la que, durante los últimos años, se ha generado una gran cantidad de parados, concretamente 5.457 .700 a finales de 2014. Entre los efectos de esta situación, a nivel psicológico, se encuentra el padecimiento de depresión (Libby y cols., 2010). Los estudios muestran que hay una relación positiva entre estar parado y tener depresión (Booker y Sacker, 2012). Así mismo, diversos estudios (Kroll, Lampert y Devitt, 2011), muestran que los desempleados tienen más problemas de salud que los empleados, y que el apoyo social facilita afrontar el desempleo. El objetivo del presente trabajo es conocer si existe diferencia en cuanto a la depresión que padecen los sujetos que se encuentran en paro y los que no lo están; asimismo nos interesa conocer el efecto que puede tener en ambas muestras el apoyo social percibido y la resiliencia. Método: Para dar respuesta al objetivo se recogió muestra de 98 sujetos que se encontraban trabajando y en paro y, de modo voluntario y anónimo, rellenaron los cuestionarios: BDI de Beck para depresión, DUKE UNK para apoyo social y la escala de resiliencia de Wagnild y Young. Resultados: Los resultados muestran altos niveles en depresión, especialmente entre la muestra de parados; no obstante, apuntan a que el efecto del apoyo social matiza y mejora los niveles de depresión entre los parados y que los parados con baja resiliencia son proclives a padecerla.

Palabras clave: parados-trabajadores, depresión, resiliencia, apoyo social, regresión.

\section{Abstract}

Introduction: Nowadays in a situation of crisis, during the last years, it has generated a lot of unemployed people, particularly 5.457.700 at the end of 2014. Among the effects of this situation, in the psychological field, it is found suffering from depression (Libby et al., 2010). Studies show that there is a positive relationship between being unemployed and being depressed (Booker \& Sacker, 2012). In that way, several studies (Kroll, Lampery and Devitt, 2011) show that the unemployed people have more 
health problems than the employed ones, and that social support facilitates facing up to unemployment. The aim of this study is to determine whether there is difference in depression suffered by subjects who are unemployed and those who are not; also we want to know the effect it can have on both samples perceived social support and resilience. Method: To be able to give an answer to the main goal, 98 samples of subjects who were working or unemployed were gathered together and voluntarily and anonymously, completed questionnaires: BDI Beck Depression, DUKE UNK for social support and resilience's scale of Wagnild and Young. Results: The results show high levels of depression, especially among the sample of unemployed people; nevertheless, they suggest that the effect of social support clarifies and improves levels of depression among the unemployed and also shows that unemployed people with low resilience are prone to suffer from it.

Keywords: Unemployed-employed, depression, resilience, social support, regression.

\section{Introducción}

Actualmente nos encontramos ante una situación de crisis en la que, durante los últimos años, se ha generado una gran cantidad de parados. A finales de 2014 se habían superado lo cinco millones; obviamente, este giro en la calidad de vida puede tener afectaciones a nivel psicológico, por lo que surge el interés en investigar si existe relación entre encontrarse desempleado y sufrir depresión.

Diversos estudios afirman que una de las causas de la depresión es estar parado (Booker y Sacker, 2012; Libby y cols., 2010). Éstos en su trabajo ponen de manifiesto la relación positiva entre estar parado y tener depresión. Así mismo, Kroll y cols. (2011), muestran que los desempleados tienen más problemas de salud que los empleados y que el apoyo social facilita afrontar el desempleo.

El objetivo del trabajo es conocer si existe diferencia en cuanto a la depresión que padecen los sujetos que se encuentran en paro y los que no lo están; así como conocer el efecto que puede tener en ambas muestras el apoyo social percibido y la resiliencia. En cuanto a las hipótesis, planteamos que:

- Los niveles de depresión serán mayores en parados que en muestra de trabajadores.

- Dentro de la muestra de parados a mayor resiliencia menor depresión.

- Dentro de la muestra de parados a mayor apoyo social menor depresión.

\section{Método}

El trabajo se realizó con un diseño ex-post-facto donde se observó la VD «Depresión», medida con el BDI de Beck y como variables independientes se midió la resiliencia con escala de Wagnild and Young y el apoyo social con el cuestionario de DUKE UNK.

Contamos con una muestra total de 98 sujetos, 67 hombres y 28 mujeres, con una media de edad de 36,85 (SD= 11,40) años. El 41,8 \% tenía cargas familiares. Del total el $75 \%$ eran personas paradas. La edad entre trabajadores y no trabajadores no resulta significativamente distinta (U-Mann Whitney $p=0,57$ ). Tampoco hay diferencias entre ser trabajador y género (coe- 
ficiente $C=0,15, p=0,13$ ), pero sí ser trabajador y tener cargas familiares (coeficiente $C=0,70$ $p=0)$, de modo que los trabajadores poseen una media inferior.

La aplicación de los cuestionarios se llevó a cabo de manera on-line, siendo la participación en el estudio totalmente voluntaria y completamente anónima. En primer lugar se recogen los datos de un grupo de parados y después se procede a pasar los cuestionarios a un número de sujetos con trabajo, con características, más o menos, similares al grupo de parados.

\section{Resultados}

Los resultados apuntan hacia diferencias significativas entre trabajadores y no trabajadores en depresión $(t=-2,98, p=0)$, los parados muestran valores más altos en depresión que los que están trabajando. También hay diferencias significativas en cuanto a apoyo social $(t=11,14, p=0)$ en este caso las medias más altas las obtienen los no parados. En cuanto a resiliencia también tienen la media más alta los no parados $(t=4,77, p=0)$. En cuanto al efecto del apoyo social y la resiliencia en la depresión de la muestra de parados, la Tabla 1 muestra los coeficientes.

Tabla 1

Pronóstico de la depresión en parados

\begin{tabular}{|c|c|c|c|c|c|c|}
\hline & & \multicolumn{2}{|c|}{$\begin{array}{l}\text { Coeficientes no } \\
\text { estandarizados }\end{array}$} & \multirow{2}{*}{$\begin{array}{c}\begin{array}{c}\text { Coeficientes } \\
\text { estandarizados }\end{array} \\
\text { Beta }\end{array}$} & \multirow[b]{2}{*}{$\mathrm{t}$} & \multirow[b]{2}{*}{ Sig } \\
\hline \multicolumn{2}{|c|}{ Model } & B & Std. error & & & \\
\hline \multirow[t]{3}{*}{1} & Constante & 39,898 & 3,003 & & 13,284 & 0,000 \\
\hline & Apoyo social & $-0,198$ & 0,081 & $-0,225$ & $-2,460$ & 0,016 \\
\hline & Resiliencia & $-0,109$ & 0,017 & $-0,602$ & $-6,596$ & 0,000 \\
\hline
\end{tabular}

a. Parados o trabajadores $=$ parados

b. Variable dependiente: resultado BDI

\section{Discusión y conclusiones}

Los resultados muestran altos niveles en depresión, especialmente entre la muestra de parados, hecho que confirma nuestra primera hipótesis.

En cuanto a la resiliencia, los resultados indican mayores valores para los trabajadores, lo que corroboraría que a mayor resiliencia también hay menos depresión.

Por último, hemos encontrado que el apoyo social favorece el decremento en depresión, ratificando nuestra tercera hipótesis.

En cuanto al pronóstico de la depresión en parados la tabla 1 muestra que ambos coeficientes: apoyo social y resiliencia, la explican significativamente y de forma inversa es decir ambas matizan y mejoran los niveles de depresión entre los parados. 


\section{Referencias bibliográficas}

Booker, C., y Sacker, A. (2012). Psychological well-being and reactions to multiple unemployment events: adaptation or sensitisation? Journal of epidemiology and community health, 66, 832-8.

Kroll, L., Lampert, T., y Devitt, C. (2011). Unemployment, social support and health problems. Deutsches Ärzteblatt International, 108, 47-52.

Libby, A., Ghushchyan, V., McQueen, R., y Campbell, J. (2010). Economic grand rounds: psychological distress and depression associated with job loss and gain: the social costs of job instability. Psychiatric services, 61, 1178-80. 